\title{
Recursos creativos de la publicidad en Internet
}

\section{Dra. María Sánchez Valle*}

Profesora de Publicidad. Universidad CEU San Pablo

\section{Dra. Belinda de Frutos Torres}

Profesora de Publicidad. Universidad CEU San Pablo

\section{Resumen:}

En este artículo se presentan los resultados de un estudio descriptivo sobre la creatividad de la publicidad en internet. Para realizar esta investigación se han analizado las creatividades de cuatro sectores representativos de estrategias publicitarias diferentes. Los resultados más importantes son que predominan las creatividades realizadas con tecnología rich media, el formato más utilizado es el banner y respecto a la posición dentro de la página web se prefiere la rotación general. Por otro lado, se pone de manifiesto que no se están utilizando solamente los formatos estandarizados por IAB Spain, lo que implica que exista una gran disparidad de tamaños.

Palabras clave:

Publicidad en internet. Formato. Recursos creativos. Rich media.

\section{Abstract:}

Within this article, the results of a descriptive study about advertising creativity on the internet are shown. To carry out this study, the creativity of four representative sectors of different advertising strategies have been analyzed. The most important result from the study are: that rich media technology is predominantly used for web-page advertisements, the most used format is banner and, with respect to the actual position

\footnotetext{
* Nota de los autores: Esta investigación ha sido realizada en el proyecto de investigación con referencia 06/HSE0105/04 financiado por la Consejería de Educación de la Comunidad de Madrid. Las autoras quieren agradecer la colaboración de Nielsen/ Net-Rating Spain en el desarrollo de este trabajo.
} 
in the web page, general rotation is preferred. On the other hand, the study showed that not only IAB Spain standard formats are being used; this implies that there are several different sizes.

Key words:

Internet advertising. Format. Creative resources. Rich media.

\section{Introducción}

Los inicios de la publicidad en Internet se remontan según Forrester Reserch (2004) a 1995 cuando se publica el primer banner en la revista Wired. Interactive Advertising Bureau Europe (2002) coincide al señalar que en 1996 se estandariza la forma, la posición y se extiende el uso del banner como formato publicitario. El siguiente paso se dará en 1998 cuando se inicia un consenso sobre el formato y la posición de una nueva forma publicitaria en Internet, el botón. Fruto de esta evolución en 2000 aparecen nuevos formatos que atienden a las nuevas necesidades y a la aparición de tecnologías como la tecnología rich media. Estos nuevos formatos ofrecen más flexibilidad, amplían las capacidades del medio y son una gran oportunidad para el desarrollo de toda la potencialidad creativa que ofrece el soporte. De esta manera, las principales claves que determinan la utilización de los diferentes recursos creativos se guían por las premisas de que los formatos grandes son mejores que los de menor tamaño, la localización de la publicidad dentro de la página es muy relevante a la hora de ser percibida por el usuario, al mismo tiempo que la tecnología rich media mejora la creatividad del medio.

Actualmente, Internet es el medio convencional que más ha crecido en el último año según los datos facilitados por INFOADEX (2006), sólo en el año 2005 la inversión en este medio se incrementó un $27,7 \%$ frente al año anterior. Por otro lado, se ha pasado de una inversión de 53,4 millones de euros en 2000 a 120,5 millones en 2005 en tan sólo cinco años (INFOADEX, 2005). Este aumento pone de manifiesto el gran potencial publicitario de Internet motivado, al mismo tiempo, por la juventud del medio y por sus propias características como la interactividad, la capacidad de personalización del mensaje, la actitud del usuario ante la publicidad en el medio, etc.

Por tanto, nos encontramos con un soporte con gran capacidad creativa, ya que, tal y como indica Interactive Advertising Bureau Spain (IAB Spain, 2004), es el medio más joven del panorama mediático $\mathrm{y}$ en el que mayor número de formatos publicitarios coexisten. Esta falta de estandarización deriva, irremediablemente, de sus características intrínsecas como son la versatilidad que ofrece, la multitud de formatos que coexisten, así como la capacidad para reflejar contenidos cada día más sofisticados. Sin embargo, esta situación no beneficia a ninguno de los actores implicados en el proceso publicitario puesto que dificulta la creación y la planificación dentro del soporte.

Por este motivo, IAB Spain realizó durante 2004 un esfuerzo para conseguir la estandarización de los formatos creativos y lo pesos de la publicidad en Internet con la finalidad de facilitar la comunicación 
entre todos los agentes implicados en la estructura del mercado publicitario -agencias, medios, anunciantes y soportes-, además de permitir un mayor conocimiento entre los actores offline.

De esta manera, la IAB Spain (2004) definió una serie de tamaños estándar para cada uno de los formatos publicitarios. Así, los tamaños definidos en píxeles por esta asociación son:

- Banner: $468 \times 60,728 \times 90$ píxeles.

- Rascacielos: $120 \times 600,160 \times 600$ píxeles.

- Robapáginas: $300 \times 250,200 \times 200$

- Botón: $120 \times 60,120 \times 90,120 \times 120,150 \times 60,150 \times 150,180 \times 150$

- Pop-up: $300 \times 250,200 \times 200,250 \times 250$

- Pop under: $300 \times 250,200 \times 200,250 \times 250$

Mientras para el resto de formatos como layer, cortinillas (interstitial), spot on line, acciones especiales (patrocionios e integraciones), formatos exclusivos y emailing, por la propia naturaleza del formato no se definen unas medidas precisas.

Sin embargo, la realidad es que no se respetan totalmente los estándares propuestos por esta asociación, aunque se empieza a vislumbrar una mayor estandarización en los formatos más tradicionales. Éste es el caso del banner estándar (468x60, 728x90 píxeles) que desde el principio ha sido el formato dominante y el estándar por excelencia (Cho, Lee y Tharp, 2001; Meland, 2000). En algunos estudios se ha demostrado cómo se configura como el formato que más reconocen los internautas cuando navegan por internet, al mismo tiempo que se considera uno de los formatos menos molestos junto con el rascacielos que la mayoría de las veces pasa desapercibido y los botones, frente a otros formatos como las ventanas рор-up o layer que se menciona como uno de los aspectos más negativos de la publicidad en Internet puesto que interfiere en la actividad del navegante, al contrario que el interstitial, formato de gran impacto que no afecta a la navegación del usuario (Gutiérrez y Frutos, 2005).

Por otro lado, unido a esta dificultad de estandarización, el lenguaje publicitario en Internet ha supuesto un avance muy significativo en sus pocos años de existencia sobre todo por el cambio experimentado en la utilización de los recursos creativos. De esta manera se ha producido la transición de un lenguaje basado en el contenido textual a un predominio de los elementos gráficos y el movimiento en las creatividades, posibilitado fundamentalmente por la utilización de la tecnología rich media (DoubleClick, 2005).

Esta innovación en la publicidad en Internet viene motivada por la capacidad de establecer una relación interactiva con el usuario, al mismo tiempo que la aplicación de la tecnología rich media permite incorporar animación, sonido, vídeo y/o interactividad a través de cualquier formato publicitario. El uso de esta tecnología está cada vez más extendido entre los sectores publicitarios con más niveles de inversión y creatividad, como por ejemplo el sector de automoción, telecomunicaciones y financiero (DoubleClick, 2005) 
Por otro lado, la evolución del lenguaje publicitario en Internet ha hecho que recursos utilizados como reclamo frecuente en un periodo determinado de la publicidad en Internet como las llamadas "haz clic" o "pulsa aquí" (Chandon, Chtourou y Fortín, 2003) sean cada vez menos empleados como elemento para atraer la atención del usuario frente a otras técnicas como la inclusión de movimiento en la propia creatividad publicitaria.

Así, en un informe sobre la publicidad en Internet elaborado por la Asociación de Usuarios de la Comunicación (AUC, 1999) se constataba la utilización de recursos expresivos que han quedado obsoletos en la actualidad, como el predominio de creatividades compuestas por una imagen estática sobre una dinámica o el mayor número de los elementos tipográficos frente a los gráficos o infográficos.

Dada esta situación, parece fundamental acometer una investigación rigurosa sobre los recursos creativos utilizados en Internet. Por tanto, a través del estudio que ahora presentamos se pretende hacer un análisis descriptivo de la publicidad en Internet con el objeto de poner de manifiesto las características formales y de contenido de la comunicación publicitaria difundida a través de este soporte. El objetivo de esta investigación se concreta en los siguientes puntos:

- Realizar un análisis descriptivo de la publicidad en Internet, teniendo en cuenta los recursos creativos más relevantes.

- Vislumbrar las principales tendencias de este ámbito de cara a un mejor aprovechamiento de la potencialidad que ofrece este medio.

\section{Procedimiento}

Para la realización de este estudio descriptivo se han analizado 422 creatividades pertenecientes a cuatro sectores publicitarios diferentes: bebidas alcohólicas -71 creatividades-, modelos de telefonía -82 creatividades-, moda (vestido y calzado) -72 creatividades- y finanzas (banca) -197 creatividades- utilizando la herramienta AdRelevance proporcionada por Nielsen/Net-Rating Spain.

La elección de estos sectores se debe a que son característicos de estrategias publicitarias diferentes y a que representan productos o servicios bien diferenciados entre sí. Otra de las razones que condicionó su elección fue que este análisis se realiza como parte de en una investigación sobre la eficacia en Internet, en la que el público elegido para la aplicación del estudio experimental eran jóvenes entre 18 y 23 años que además corresponden con el perfil mayoritario del usuario en Internet. De esta manera las creatividades seleccionadas corresponden a artículos funcionales -telefonía y finanzas- y expresivos -bebidas alcohólicas y moda-, al mismo tiempo que son productos que representan para el usuario estrategias de alta implicación -telefonía y moda- y baja implicación -bebidas alcohólicas y financiero-.

Por otro lado, estos sectores eran idóneos para realizar este estudio ya que desde el punto de vista publicitario presentan una gran actividad publicitaria y creativa (INFOADEX, 2006). 
Por tanto, se realizó un análisis exhaustivo de todas las creatividades que habían aparecido en Internet desde el 1 de enero hasta el 30 de octubre de $2005^{1}$. Pormenorizadamente las fechas de las campañas objeto de estudio son las siguientes:

- Bebidas alcohólicas: 1 de enero al 27 de septiembre de 2005.

- Financiero: 1 de enero al 25 de octubre de 2005.

- Telefonía: 1 de enero al 6 de octubre de 2005.

- Moda: 1 de enero al 12 de octubre de 2005.

Las creatividades seleccionadas fueron analizadas teniendo en cuenta una serie de parámetros que se pueden englobar en diferentes categorías:

- Descripción técnica de la creatividad: sector, campaña, producto, marca y periodo de vigencia.

- Ubicación de la creatividad: categoría del soporte donde se emplaza la publicidad y posición dentro de la página.

- Características formales: animación, tipo de animación, interactividad, movimiento, existencia de un enlace y formato, tamaño.

- Características de contenido: tipo de campaña, marca asociada. existencia de ilustración, tipo de ilustración, número de colores, color predominante, tipografía destacada, idioma, eslogan, logotipo, datos de contacto, sonido, fórmula pregunta, reclama clic y respeta el deseo del usuario.

\section{Resultados}

A continuación, pasamos a exponer los principales resultados agrupados en torno a las variables que hemos descrito en el epígrafe anterior.

\subsection{Descripción técnica de la creatividad}

Dentro de los sectores analizados, el ámbito que destaca por su actividad publicitaria en Internet es, sin lugar a dudas, el sector financiero, que representa casi la mitad de los anuncios analizados (46,7\%). Estas creatividades corresponden a 56 anunciantes que realizaron 66 campañas diferentes.

Respecto al resto de los sectores, el número de creatividades se reparte de una manera bastante homogénea. En cada uno de ellos se detectaron entre 82 y 71 creatividades diferentes. Hay que señalar que en el caso de las bebidas alcohólicas estas creatividades corresponden a 30 anunciantes diferentes que realizaron 28 campañas distintas, mientras en el sector de la telefonía son 13 los anunciantes y 36

\footnotetext{
${ }^{1}$ Excluyendo la autopromoción.
} 
las campañas emitidas, frente a los 28 anunciantes de moda que llevaron a cabo 31 campañas. En cuanto al periodo de vigencia de las campañas, la mayoría tenían una duración media situada entre dos semanas y un mes, aproximadamente.

Los emisores en todos los casos corresponden a los principales anunciantes en medios convencionales. Así, en el sector financiero destaca ING Direct; bebidas alcohólicas J\&B; telefonía Nokia y moda, Nike y Adidas.

Tabla 1

Distribución de la publicidad por sectores

\begin{tabular}{|l|c|c|}
\hline \multicolumn{1}{|c|}{ Sector } & Frecuencia & Porcentaje \\
\hline Finanzas (Banca) & 197 & 46,7 \\
\hline Modelos Telefonía & 82 & 19,4 \\
\hline Moda: vestido y calzado & 72 & 17,1 \\
\hline Bebidas alcohólicas & 71 & 16,8 \\
\hline Total & 422 & 100,0 \\
\hline
\end{tabular}

Como se puede apreciar en la tabla 2, los servicios bancarios son los productos sobre los que se realizan más piezas publicitarias (37\%), seguido a bastante distancia de los teléfonos móviles $(11,4 \%)$, moda $(9 \%)$ y cerveza $(8,8 \%)$.

Tabla 2

Distribución por producto

\begin{tabular}{|l|c|c|}
\hline \multicolumn{1}{|c|}{ Producto } & Frecuencia & Porcentaje \\
\hline Servicio bancario & 156 & 37,0 \\
\hline Teléfono móvil & 48 & 11,4 \\
\hline Moda & 38 & 9,0 \\
\hline Cerveza & 37 & 8,8 \\
\hline Bebidas alta gradación & 26 & 6,2 \\
\hline Calzado & 25 & 5,9 \\
\hline Promoción & 23 & 5,5 \\
\hline Concurso & 20 & 4,7 \\
\hline Otros & 49 & 11,5 \\
\hline Total & 422 & 100,0 \\
\hline
\end{tabular}




\section{2. $\quad$ Ubicación de la creatividad}

Un aspecto tan importe como la propia creatividad de la campaña, es la ubicación de la pieza publicitaria en función de dos parámetros; por un lado, en qué tipo de página web se encuentra la creatividad y, por otro, qué posición ocupa dentro de la página.

En relación a la categoría del soporte en el que se emplaza la publicidad, la mayoría se ubican en soportes que ofrecen información y noticias $(41,7 \%)$, seguido de los portales, buscadores y comunidades $(27,5 \%)$ y sitios editoriales $(14,9 \%)$. Por tanto, aquellos soportes en los que se ofrece una información de interés general son, precisamente, los que más publicidad acogen entre sus páginas.

Tabla 3

Distribución por soporte

\begin{tabular}{|l|c|c|c|c|c|}
\hline \multicolumn{1}{|c|}{ Soporte } & $\begin{array}{c}\text { Bebidas } \\
\text { alcohólicas }\end{array}$ & Financiero & Telefonía & Moda & Total \\
\hline Información y noticias & 28,3 & 53,8 & 25,6 & 40,3 & $\mathbf{4 1 , 7}$ \\
\hline Portales/ Buscadores y comunidades & 28,2 & 22,8 & 39 & 26,4 & $\mathbf{2 7 , 5}$ \\
\hline Sitios editoriales & 19,7 & 9,1 & 25,6 & 13,9 & $\mathbf{1 4 , 9}$ \\
\hline Edición & 9,9 & 4,1 & 2,4 & 9,7 & $\mathbf{5 , 7}$ \\
\hline Distribución/ Comercio electrónico & 9,9 & 1 & - & 4,2 & $\mathbf{2 , 8}$ \\
\hline Institucional, asociaciones y eventos & 1,4 & 3 & 1,2 & 2,8 & $\mathbf{2 , 4}$ \\
\hline Inmobiliario & - & 3 & 1,2 & 1,4 & $\mathbf{1 , 9}$ \\
\hline Empleo & - & 2 & 1,2 & - & $\mathbf{1 , 2}$ \\
\hline Transportes/ Turismo & 2,8 & - & 1 & 1,4 &, $\mathbf{7}$ \\
\hline Telefonía/ Telecomunicaciones & - & - & 2,4 & - &, $\mathbf{5}$ \\
\hline Servicios empresariales & - & - & 1,2 & - &, $\mathbf{2}$ \\
\hline Ocio/ entretenimiento & - & 0,5 & - & - & $\mathbf{, 2}$ \\
\hline Finanzas (banca) & - & 0,5 & - & - &, $\mathbf{2}$ \\
\hline
\end{tabular}

Respecto a la posición de la creatividad dentro de la página, el 91,9\% de las creatividades presentan una rotación general dentro del conjunto de la página, es decir, las piezas publicitarias no tienen una ubicación fija. Este comportamiento es más acusado en el sector financiero, telefonía y moda, donde todas las creatividades rotan en el interior del soporte. Sólo en el sector de las bebidas alcohólicas casi la mitad de la publicidad se ubica en posiciones fijas, repartiéndose estas situaciones, como puede apreciarse en la tabla 4, de una manera uniforme entre las posiciones: media centro, derecha o izquierda y superior centro, derecha o izquierda. 
Este comportamiento descubre que los anunciantes están aprovechando una de las ventajas que ofrece la publicidad en Internet, que no es otra que la capacidad de alternar las posiciones para aumentar las posibilidades de que el navegante vea la publicidad que aparece en la página, ya que hay ciertas lugares dentro de la web que no reciben tanta la atención por parte de los usuarios como son la parte superior o inferior de la página, frente a otros como interior de la página (Frutos y Sánchez, en prensa).

Tabla 4

Posición dentro de la página web

\begin{tabular}{|l|c|c|c|c|c|}
\hline \multicolumn{1}{|c|}{ Posición } & $\begin{array}{c}\text { Bebidas } \\
\text { alcohólicas }\end{array}$ & Financiero & Telefonía & Moda & Total \\
\hline Rotación general & 52,1 & 100 & 100 & 100 & $\mathbf{9 1 , 9}$ \\
\hline Media derecha & 14,1 & - & - & - & $\mathbf{2 , 4}$ \\
\hline Media centro & 11,3 & - & - & - & $\mathbf{1 , 9}$ \\
\hline Superior centro & 11,3 & - & - & - & $\mathbf{1 , 9}$ \\
\hline Otros $^{2}$ & 11,2 & - & - & - & \\
\hline
\end{tabular}

\subsection{Características formales}

Respecto a la tecnología aplicada a las creatividades, se impone la utilización de los recursos que se engloban bajo la denominación rich media, prácticamente la totalidad de las creatividades se realizan con flash o gif animado, lo que realmente consigue diferenciar claramente este soporte respecto al resto de medios convencionales ya que esta tecnología aporta a la publicidad sofisticados recursos expresivos que en otros medios no se pueden conseguir.

Tabla 5

Tecnología aplicada en la publicidad

\begin{tabular}{|l|c|c|c|c|c|}
\hline \multicolumn{1}{|c|}{ Tecnología } & $\begin{array}{c}\text { Bebidas } \\
\text { alcohólicas }\end{array}$ & Financiero & Telefonía & Moda & Total \\
\hline Flash & 54,9 & 39,1 & 52,4 & 58,3 & $\mathbf{4 7 , 6}$ \\
\hline Gif animado & 39,4 & 55,3 & 28 & 23,6 & $\mathbf{4 1 , 9}$ \\
\hline Gif estático & 5,6 & 5,1 & 19,5 & 18,1 & $\mathbf{1 0 , 4}$ \\
\hline
\end{tabular}

\footnotetext{
${ }^{2}$ Media izquierda, superior derecha y superior izquierda.
} 
Estrechamente relacionado con la tecnología aplicada, se encuentra la existencia de animación en las creatividades. De esta manera el $88,9 \%$ de las creatividades son dinámicas. Así, en más de la mayoría de las creatividades $(67,2 \%)$ los elementos animados son las ilustraciones y el texto, porcentaje es aún mayor en el sector de las bebidas alcohólicas (80\%) y la telefonía $(80,3 \%)$.

Tabla 6

Uso y tipo de animación

\begin{tabular}{|l|c|c|c|c|c|}
\hline \multicolumn{1}{|c|}{ Animación } & $\begin{array}{c}\text { Bebidas } \\
\text { alcohólicas }\end{array}$ & Financiero & Telefonía & Moda & Total \\
\hline Dinámico & 91,5 & 94,4 & 80,5 & 80,6 & $\mathbf{8 8 , 9}$ \\
\hline Estático & 8,5 & 5,6 & 19,5 & 19,4 & $\mathbf{1 1 , 1}$ \\
\hline Tipo animación & & & & & \\
\hline Ilustración y texto & 80,0 & 59,7 & 80,3 & 62,1 & $\mathbf{6 7 , 2}$ \\
\hline Sólo texto & 16,9 & 37,1 & 18,2 & 31,0 & $\mathbf{2 9 , 3}$ \\
\hline Sólo ilustración & 3,1 & 1,6 & 1,5 & 6,9 & $\mathbf{2 , 7}$ \\
\hline Sólo color & - & 1,6 & - & - &, $\mathbf{8}$ \\
\hline
\end{tabular}

Si bien señalamos que la tecnología rich media permite la interactividad del usuario con la publicidad y, como se ha reflejado la mayoría de las creatividades utilizan esta tecnología, sorprendentemente el 90,5\% de los anuncios no son interactivos, es decir, no se utilizan como canal para recoger las respuestas del navegante.

Sin embargo, lo que sí ofrece mayoritariamente la publicidad en Internet es la posibilidad de obtener más información sobre el producto o servicio ofertado en el mismo momento de la navegación a través de la inclusión de un enlace, de tal manera que en todas ellas existe un enlace que nos remite en el $77,5 \%$ de los casos a un site genérico -un $89,8 \%$ en financiero y un $82 \%$ en moda- un $18,7 \%$ a un microsite -un $35,2 \%$ en las bebidas alcohólicas- y un 3,8\% permiten una navegación dentro del site del soporte.

Tabla 7

Interactividad y enlace

\begin{tabular}{|l|c|c|c|c|c|}
\hline \multicolumn{1}{|c|}{ Interactividad } & $\begin{array}{c}\text { Bebidas } \\
\text { alcohólicas }\end{array}$ & Financiero & Telefonía & Moda & Total \\
\hline No son interactivas & 81,7 & 93,4 & 89 & 95,8 & $\mathbf{9 0 , 5}$ \\
\hline Sí son interactivas & 18,3 & 6,6 & 11 & 4,2 & $\mathbf{9 , 5}$ \\
\hline
\end{tabular}




\begin{tabular}{|l|c|c|c|c|c|}
\hline \multicolumn{1}{|c|}{ Interactividad } & $\begin{array}{c}\text { Bebidas } \\
\text { alcohólicas }\end{array}$ & Financiero & Telefonía & Moda & Total \\
\hline Enlace a & & & & & \\
\hline Site genérico & 53,5 & 89,8 & 64,6 & 82 & $\mathbf{7 7 , 5}$ \\
\hline Microsite & 35,2 & 9,6 & 31,7 & 12,5 & $\mathbf{1 8 , 7}$ \\
\hline Navegación dentro del site del soporte & 11,3 & 0,5 & 3,7 & 5,6 & $\mathbf{3 , 8}$ \\
\hline
\end{tabular}

Dentro de las características formales, el aspecto más importante es el formato que reviste el mensaje publicitario. El banner es el más utilizado en todos los sectores (41\%), seguido del robapáginas (23,3\%), excepto en el sector financiero donde es el rascacielos el formato que ocupa la segunda posición. En tercer lugar se sitúa el botón (11,6\%), excepto en el sector de financiero que se encuentra en segunda posición.

Esta utilización del banner por encima del resto de los formatos puede deberse a que tradicionalmente ha sido el formato que más se ha empleado (AUC, 2000) en Internet y uno de los primeros formatos estandarizados por IAB (2004). Al mismo tiempo que el banner junto con el rascacielos es considerado por los usuarios como la publicidad menos intrusiva (Frutos y Gutiérrez, 2005). Sin embargo, los estudios demuestran que no es el formato que posibilita un mayor recuerdo de la publicidad, sino que otras formas publicitarias como el botón se muestran más efectivas (Frutos y Sánchez, en prensa).

Por otro lado, los resultados que se observan en esta tabla ponen de manifiesto que aún no se están explotando todas las posibilidades tecnológicas que ofrece Internet. Prueba de ello es la escasa utilización de otros formatos más novedosos como el spot online; al mismo tiempo que se reafirma la tendencia a evitar los formatos que interfieren en la navegación del usuario como los layers (Gutiérrez y Frutos, 2005)

Tabla 8

Formato de la publicidad

\begin{tabular}{|l|c|c|c|c|c|}
\hline \multicolumn{1}{|c|}{ Formato } & $\begin{array}{c}\text { Bebidas } \\
\text { alcohólicas }\end{array}$ & Financiero & Telefonía & Moda & Total \\
\hline Banner & 42,3 & 44,2 & 36,6 & 36,1 & $\mathbf{4 1 , 0}$ \\
\hline Robapáginas & 25,4 & 18,3 & 26,8 & 30,6 & $\mathbf{2 3 , 2}$ \\
\hline Botón & 11,3 & 8,1 & 17,1 & 15,3 & $\mathbf{1 1 , 6}$ \\
\hline Rascacielos & 7 & 24,4 & 13,4 & 9,7 & $\mathbf{1 6 , 8}$ \\
\hline Interstitial & 5,6 & 5,1 & 1,2 & 1,4 & $\mathbf{3 , 8}$ \\
\hline Layer & 2,8 & - & 3,7 & 2,8 & $\mathbf{1 , 7}$ \\
\hline Spot online & 5,6 & - & - & 4,2 & $\mathbf{1 , 7}$ \\
\hline Patrocinio & - & - & 1,2 & - &, $\mathbf{2}$ \\
\hline
\end{tabular}


Relacionado con el formato se encuentra el tamaño de las creatividades, ante la disparidad de extensión que presentan los diferentes formatos publicitarios -se han encontrado 87 tamaños diferentesse ha procedido a reflejar los diez tamaños más frecuente. Con el mismo objetivo, se ha tenido en cuenta únicamente el tamaño de la primera pieza publicitaria que ofrece la herramienta AdRelevance.

De estos diez tamaños, seis corresponden a formatos estandarizados por IAB Spain. El área más utilizada se corresponde con el formato más utilizado (banner), el segundo con el robapáginas, el cuarto con el banner, el quinto con el robapáginas y el sexto con el botón. Es sintomático de esta falta de estandarización que la tercera área de superficie no corresponda a ningún tamaño estandarizado. Por tanto, se aprecia que, a pesar de la disparidad de tamaños, hay una cierta estandarización en los tamaños de los formatos más frecuentes como el banner, botón o robapáginas aunque también se pueden encontrar otros tamaños dentro de estos formatos que conviven con las áreas estandarizadas por IAB Spain (2002).

Por otro lado, no hay grandes diferencias entre la utilización de los diferentes tamaños de la creatividad en los sectores analizados, a excepción del caso del área 1200x400 que no responde a ninguno de los formatos estandarizados IAB Spain, y que aparece en un 18,3\% de las creatividades del sector financiero.

Tabla 9

Tamaño de la publicidad

\begin{tabular}{|l|c|c|c|c|c|}
\hline \multicolumn{1}{|c|}{ Área píxeles } & $\begin{array}{c}\text { Bebidas } \\
\text { alcohólicas }\end{array}$ & Financiero & Telefonía & Moda & Total \\
\hline $\mathbf{4 6 8} \times \mathbf{6 0}$ & 25,4 & 27,9 & 22 & 15,3 & $\mathbf{2 4 , 2}$ \\
\hline $\mathbf{3 0 0} \times \mathbf{2 5 0}$ & 16,9 & 10,7 & 20,7 & 19,4 & $\mathbf{1 5 , 2}$ \\
\hline $\mathbf{1 2 0} \times \mathbf{4 0 0}$ & - & 18,3 & - & - & $\mathbf{8 , 5}$ \\
\hline $\mathbf{7 2 8} \times \mathbf{9 0}$ & 1,4 & 6,1 & 6,1 & 9,7 & $\mathbf{5 , 9}$ \\
\hline $\mathbf{2 0 0} \times \mathbf{2 0 0}$ & 9,9 & 4,1 & 1,2 & 6,9 & $\mathbf{5}$ \\
\hline $\mathbf{1 2 0} \times \mathbf{6 0 0}$ & 0 & 4,6 & 8,5 & 4,2 & $\mathbf{4 , 5}$ \\
\hline $\mathbf{7 5 0} \times \mathbf{1 0 0}$ & 9,9 & 0,5 & 3,7 & 9,7 & $\mathbf{4 , 3}$ \\
\hline $\mathbf{1 6 0} \times \mathbf{6 0 0}$ & 2,8 & 0,5 & 3,7 & 4,2 & $\mathbf{2 , 2}$ \\
\hline $\mathbf{8 0 0} \times \mathbf{6 0 0}$ & 2,8 & 3 & 1,2 & - & $\mathbf{2 , 1}$ \\
\hline $\mathbf{3 0 0} \times \mathbf{3 0 0}$ & 1,4 & 0,5 & 0 & 4,2 & $\mathbf{1 , 2}$ \\
\hline Resto & 29,5 & 23,8 & 32,9 & 26,4 & $\mathbf{2 6 , 9}$ \\
\hline
\end{tabular}


Dada la importancia de la falta de estandarización de los diferentes formatos publicitarios, a continuación se analizan los tamaños que presentan los formatos publicitarios estandarizados por IAB Spain.

\subsubsection{Banner}

El banner es el formato publicitario de Internet más antiguo, estandarizado en 1996. Este formato está ubicado en la parte superior de las páginas web y puede usar tecnología gif, animado, flah o jpeg. Hay dos tamaños de banner estandarizados por IAB Spain (2004): $468 \times 60$ píxeles y $728 \times 90$ píxeles, con un peso máximo permitido de 15k.

Tabla 10

Área de superficie del banner expresada en frecuencias absolutas

\begin{tabular}{|c|c|c|c|c|c|}
\hline Área píxeles & $\begin{array}{l}\text { Bebidas } \\
\text { alcohólicas }\end{array}$ & Financiero & Telefonía & Moda & Total \\
\hline $220 \times 59$ & & 1 & & & 1 \\
\hline $230 \times 65$ & & 1 & & & 1 \\
\hline $240 \times 60$ & & 1 & & & 1 \\
\hline $245 \times 90$ & & 2 & & & 2 \\
\hline $297 \times 26$ & & 1 & & & 1 \\
\hline $300 \times 50$ & & 1 & & & \\
\hline $300 \times 55$ & & & 1 & & 1 \\
\hline $300 \times 200$ & & 3 & & & 3 \\
\hline $300 \times 250$ & 1 & & 1 & & 2 \\
\hline $320 \times 55$ & & & 1 & & 1 \\
\hline $392 \times 94$ & & & 1 & & 1 \\
\hline $458 \times 60$ & 2 & & & & 2 \\
\hline $468 \times 35$ & & & & 1 & 1 \\
\hline $468 \times 60$ & 18 & 55 & 18 & 11 & 102 \\
\hline $600 \times 25$ & & 1 & & & 1 \\
\hline
\end{tabular}




\begin{tabular}{|l|c|c|c|c|c|}
\hline \multicolumn{1}{|c|}{ Área píxeles } & $\begin{array}{c}\text { Bebidas } \\
\text { alcohólicas }\end{array}$ & Financiero & Telefonía & Moda & Total \\
\hline $\mathbf{7 2 0} \times \mathbf{3 0 0}$ & 1 & 1 & & & 1 \\
\hline $\mathbf{7 2 8} \times \mathbf{9 0}$ & & 12 & 5 & 7 & 1 \\
\hline $\mathbf{7 2 8} \times \mathbf{9 0}$ & 7 & & 3 & & 10 \\
\hline $\mathbf{7 5 0} \times \mathbf{1 0 0}$ & & 1 & & & 1 \\
\hline $\mathbf{7 3 0} \times \mathbf{1 8 0}$ & 1 & 3 & & & 3 \\
\hline $\mathbf{7 3 0} \times \mathbf{9 0}$ & & 1 & & & \\
\hline $\mathbf{7 5 0} \times \mathbf{9 0}$ & & & 1 & & 3 \\
\hline $\mathbf{7 5 0} \times \mathbf{1 0 0}$ & & 1 & & & 1 \\
\hline $\mathbf{7 7 0} \times \mathbf{6 5}$ & & 2 & & & 2 \\
\hline $\mathbf{7 7 0} \times \mathbf{9 0}$ & $\mathbf{3 0}$ & $\mathbf{8 7}$ & $\mathbf{3 1}$ & $\mathbf{2 6}$ & $\mathbf{1 7 4}$ \\
\hline $\mathbf{7 8 0} \times \mathbf{9 0}$ & & & & & \\
\hline Total & & & & & \\
\hline
\end{tabular}

El 73,5\% de los banners analizados utilizan alguno de los dos formatos estandarizados por IAB Spain, 102 banners, lo que supone que el 59\% del total, tienen un tamaño de $468 \times 60$ píxeles y 24 banners (el $14,5 \%$ ) están basados en el tamaño $728 \times 90$. Aunque es el formato en el que más se utilizan los formatos estandarizados no se puede dejar de señalar que un $26,5 \%$ de los banners no responden a ninguna de estas superficies estándar. Por sectores, el caso más llamativo es el de las bebidas alcohólicas en el que 7 banners -un 23,3\%- presentan un tamaño de $750 \times 100$ píxeles, y en el sector moda 7 banners -un 26,9\% - tienen unas dimensiones de $750 \times 100$ píxeles.

De un total de 174 banners, se han registrado 26 tamaños diferentes. Respecto a la longitud de este formato, hay muy pocos banners cuyos píxeles horizontales sean inferiores a 300, al mismo tiempo que la mayoría presentan un ancho entre 60 y 90 píxeles.

\subsubsection{Robapáginas}

El robapáginas es publicidad que se inserta en la parte inferior de la página. IAB Spain ha estandarizado este formato con dos dimensiones: $300 \times 250$ píxeles y $200 \times 200$ píxeles. La creatividad puede usar tecnología gif, flash o jpeg. El peso máximo permitido del formato es de 20k. 
Tabla 11

Área de superficie del robapáginas expresada en frecuencias absolutas

\begin{tabular}{|l|c|c|c|c|c|}
\hline \multicolumn{1}{|c|}{ Área píxeles } & $\begin{array}{c}\text { Bebidas } \\
\text { alcohólicas }\end{array}$ & Financiero & Telefonía & Moda & Total \\
\hline $\mathbf{1 4 0} \times \mathbf{1 4 0}$ & & 2 & 1 & & 3 \\
\hline $\mathbf{1 5 0} \times \mathbf{1 5 0}$ & & 1 & & & 1 \\
\hline $\mathbf{1 6 0} \times \mathbf{1 6 0}$ & & 1 & & & 1 \\
\hline $\mathbf{1 9 4} \times \mathbf{1 9 4}$ & 1 & & 1 & & 2 \\
\hline $\mathbf{2 0 0} \times \mathbf{2 0 0}$ & 6 & 8 & 1 & 5 & 20 \\
\hline $\mathbf{2 9 0} \times \mathbf{1 4 0}$ & & & 1 & & 1 \\
\hline $\mathbf{5 0} \times \mathbf{1 0 0}$ & 1 & & & & 1 \\
\hline $\mathbf{3 0 0} \times \mathbf{2 5 0}$ & 9 & 20 & 15 & 3 & 4 \\
\hline $\mathbf{3 0 0} \times \mathbf{3 0 0}$ & & 1 & & & 1 \\
\hline $\mathbf{3 2 1} \times \mathbf{1 7 5}$ & & 1 & & & 1 \\
\hline $\mathbf{3 5 0} \times \mathbf{3 5 0}$ & & & 1 & & 1 \\
\hline $\mathbf{4 0 0} \times \mathbf{3 0 0}$ & 1 & & & & 1 \\
\hline $\mathbf{4 3 0} \times \mathbf{2 3 8}$ & & & 1 & & 2 \\
\hline $\mathbf{4 5 0} \times \mathbf{4 5 0}$ & & 1 & & & 1 \\
\hline $\mathbf{7 2 5} \times \mathbf{9 0}$ & & & & & \\
\hline Total & 18 & 35 & 22 & 22 & 97 \\
\hline
\end{tabular}

En el caso del robapáginas prácticamente todas las creatividades responden a los formatos estandarizados por IAB Spain. El más utilizado es el de $300 \times 250$ píxeles en 57 piezas -un 58,3\%-, frente a 20 creatividades -un $20,4 \%$ - que tienen un formato de $200 \times 200$ píxeles. No se encuentran diferencias importantes entre los sectores analizados.

Se han encontrado 15 tamaños diferentes respecto a un total de 97 robapáginas. Como norma general, los píxeles horizontales se sitúan entre los 200 y 400 y los verticales entre los 100 y 300 píxeles.

\subsubsection{Rascacielos}

El rascacielos o skyscreaper es un formato publictario de dimensión vertical que puede utilizar tecnología gif, flash o jpeg y cuyo peso máximo de la creatividad es $20 \mathrm{k}$. Los tamaños estandarizados por IAB Spain (2004) para este formato son $120 \times 600$ píxeles y $160 \times 600$ píxeles. 
Tabla 12

Área de superficie del rascacielos expresada en frecuencias absolutas

\begin{tabular}{|c|c|c|c|c|c|}
\hline Área píxeles & $\begin{array}{l}\text { Bebidas } \\
\text { alcohólicas }\end{array}$ & Financiero & Telefonía & Moda & Total \\
\hline $100 \times 300$ & & & & 2 & 2 \\
\hline $100 \times 600$ & & & 1 & 1 & 2 \\
\hline $120 \times 240$ & & 1 & & & 1 \\
\hline $120 \times 400$ & & 36 & & & 36 \\
\hline $120 \times 600$ & & 9 & 6 & 3 & 18 \\
\hline $120 \times 200$ & 1 & & & & 1 \\
\hline $120 \times 240$ & & 1 & & & 1 \\
\hline $140 \times 400$ & 2 & & 1 & & 3 \\
\hline $160 \times 600$ & 2 & 1 & 3 & 1 & 7 \\
\hline $300 \times 250$ & & 1 & & & 1 \\
\hline Total & 5 & 49 & 11 & 7 & 72 \\
\hline
\end{tabular}

En total se han registrado diez tamaños diferentes de un total de 72 rascacielos, de ellos sólo 25 corresponden a los formatos estandarizado por IAB Spain. El tamaño de 18 rascacielos -el 25,4\% del total- es de $120 \times 600$ píxeles y 7 piezas -el 9,9\%- tienen una dimensión de $160 \times 600$ píxeles.

Dentro del sector financiero, 36 rascacielos -el 50,7\% del total de los rascacielos analizados- tienen una resolución de $120 \times 400$ píxeles que no corresponde con ninguno de los formatos estándar.

En general, la mayoría de los rascacielos tienen una medidas entre 100 y 160 píxeles horizontales y entre 400 y 600 píxeles verticales.

\subsubsection{Botón}

Los botones son un tipo de creatividad en la que sí se hace clic sobre ellos, dirige al usuario a la página web del anunciante. La creatividad puede usar la tecnología gif, flash o jpeg. El peso máximo permitido del formato es $8 \mathrm{k}$. los botones estandarizados por IAB Spain tienen seis dimensiones $120 \times 60,120 \times$ $90,120 \times 120,150 \times 60,150 \times 150,180 \times 150$ píxeles. 
Tabla 13

Área de superficie del botón

\begin{tabular}{|c|c|c|c|c|c|}
\hline Área píxeles & $\begin{array}{c}\text { Bebidas } \\
\text { alcohólicas }\end{array}$ & Financiero & Telefonía & Moda & Total \\
\hline $60 \times 45$ & & & 1 & & 1 \\
\hline $90 \times 90$ & 1 & 1 & 2 & & 4 \\
\hline $95 \times 95$ & & 1 & & & 1 \\
\hline $100 \times 60$ & & 1 & & & 1 \\
\hline $120 \times 30$ & & & & 2 & 2 \\
\hline $120 \times 60$ & 1 & & 2 & 1 & 4 \\
\hline $120 \times 600$ & & & 1 & & 1 \\
\hline $120 \times 80$ & 1 & & 1 & & 2 \\
\hline $125 \times 30$ & & & 1 & & 1 \\
\hline $130 \times 40$ & & & & 1 & 1 \\
\hline $138 \times 89$ & & 1 & & & 1 \\
\hline $139 \times 90$ & & & 2 & & 2 \\
\hline $140 \times 90$ & & 2 & & & 2 \\
\hline $140 \times 100$ & & 1 & & & 1 \\
\hline $145 \times 41$ & & & & 1 & 1 \\
\hline $150 \times 50$ & & 1 & 1 & & 2 \\
\hline $150 \times 80$ & & & 1 & & 1 \\
\hline $155 \times 51$ & & 1 & & & 1 \\
\hline $160 \times 70$ & 2 & & & & 2 \\
\hline $185 \times 185$ & 1 & 1 & & & 2 \\
\hline $195 \times 90$ & & & 1 & & 1 \\
\hline $199 \times 60$ & & & 1 & & 1 \\
\hline $200 \times 200$ & 1 & & & & 1 \\
\hline $200 \times 57$ & 1 & & & & 1 \\
\hline $200 \times 60$ & & 1 & & & 1 \\
\hline $220 \times 56$ & & & & 1 & 1 \\
\hline $225 \times 100$ & & & & 2 & 2 \\
\hline $234 \times 60$ & & & & 2 & 2 \\
\hline
\end{tabular}

174 doxa comunicación n. ${ }^{\circ} 5$ 


\begin{tabular}{|l|c|c|c|c|c|}
\hline \multicolumn{1}{|c|}{ Área píxeles } & $\begin{array}{c}\text { Bebidas } \\
\text { alcohólicas }\end{array}$ & Financiero & Telefonía & Moda & Total \\
\hline $\mathbf{2 4 0} \times \mathbf{9 0}$ & & 1 & & & 1 \\
\hline $\mathbf{2 4 5} \times \mathbf{9 0}$ & & 2 & & & 2 \\
\hline $\mathbf{2 5 0} \times \mathbf{1 0 0}$ & 1 & & & & 1 \\
\hline $\mathbf{3 0 0} \times \mathbf{5 0}$ & & & & 1 & 1 \\
\hline $\mathbf{4 1 7} \times \mathbf{4 0 0}$ & 1 & & & & 1 \\
\hline Total & 10 & 14 & 14 & 11 & 49 \\
\hline
\end{tabular}

Si existe un formato en el que no hay una estandarización es precisamente en el caso de los botones, donde de los 49 botones analizados se han encontrado 33 áreas de superficie diferentes, A esta situación caótica se añade que sólo el $8,2 \%$ de todos los botones, lo que corresponde a 4 botones, presentan el tamaño estandarizado por IAB de $120 \times 60$ píxeles. Si atendemos a los resultados que presentan los diferentes sectores, se llega a la conclusión de que no hay una uniformidad a la hora de crear piezas publicitarias en este formato.

En general, el número de píxeles horizontales suele ser inferior a 200, mientras los píxeles verticales no superan los 150 .

\subsubsection{Interstitial}

Las cortinillas o interstitials son formatos que se muestran como una ventana completa para presentar su mensaje. Al ser anuncios de corta duración para que el usuario llegue al destino, resultan muy eficaces para llamar su atención. Con un peso de 30k, no existe ningún tamaño estandarizado por IAB Spain.

Tabla 14

Área de superficie del interstitial expresada en frecuencias absolutas

\begin{tabular}{|l|c|c|c|c|c|}
\hline \multicolumn{1}{|c|}{ Área píxeles } & $\begin{array}{c}\text { Bebidas } \\
\text { alcohólicas }\end{array}$ & Financiero & Telefonía & Moda & Total \\
\hline $\mathbf{4 0 0} \times \mathbf{6 0 0}$ & & 1 & & & 1 \\
\hline $\mathbf{4 6 8} \times \mathbf{3 9 0}$ & 1 & & & & 1 \\
\hline $\mathbf{5 0 0} \times \mathbf{5 0 0}$ & & 2 & & & 2 \\
\hline $\mathbf{6 0 0} \times \mathbf{4 0 0}$ & & 1 & & & 1 \\
\hline $\mathbf{6 1 3} \times \mathbf{4 6 0}$ & 1 & & & & 1 \\
\hline $\mathbf{8 0 0} \times \mathbf{6 0 0}$ & 2 & 6 & 1 & & 9 \\
\hline $\mathbf{1 . 2 0 0} \times \mathbf{7 6 8}$ & & & & 1 & 1 \\
\hline Total & 4 & 10 & 1 & 1 & 16 \\
\hline
\end{tabular}


Se han encontrado 7 tamaños diferentes de un total de 16 cortinillas analizadas, aunque IAB no ha estandarizado ningún tamaño más de la mitad, 9 casos, lo que supone un $56,3 \%$, tienen unas dimensiones de $800 \times 600$ píxeles. Como norma general, el tamaño se sitúa entre los 400 y 800 píxeles horizontales y los 400 y 600 píxeles verticales.

\subsubsection{Layer}

El layer es un formato publicitario que se despliega y se mueve por toda la pantalla gracias a su tecnología, superponiéndose al contenido de la página. Puede partir o no de un formato integrado. Los banners desplegables se incluyen dentro de esta categoría. Su peso es de $30 \mathrm{k}$.

Tabla 15

Área de superficie del layer expresada en frecuencias absolutas

\begin{tabular}{|l|c|c|c|c|c|}
\hline \multicolumn{1}{|c|}{ Área píxeles } & $\begin{array}{c}\text { Bebidas } \\
\text { alcohólicas }\end{array}$ & Financiero & Telefonía & Moda & Total \\
\hline $\mathbf{1 6 0} \times \mathbf{6 0 0}$ & & & & 1 & 1 \\
\hline $\mathbf{3 0 0} \times \mathbf{3 0 0}$ & 1 & & & & 1 \\
\hline $\mathbf{3 0 0} \times \mathbf{2 5 0}$ & & & 1 & & 1 \\
\hline $\mathbf{4 0 0} \times \mathbf{4 0 0}$ & & & 1 & 1 & 2 \\
\hline $\mathbf{6 9 9} \times \mathbf{2 7 8}$ & 1 & & & & 1 \\
\hline $\mathbf{7 0 0} \times \mathbf{2 7 0}$ & & & 1 & & 1 \\
\hline Total & 2 & & 3 & 2 & 7 \\
\hline
\end{tabular}

En total se han localizado siete layers, aunque IAB Spain no ha estandarizado este formato. Las medidas se encuentran entre los 300 y 700 píxeles horizontales y los 200 y 400 píxeles verticales. Entre los layers encontrados se han hallado 7 tamaños diferentes.

\subsubsection{Otros formatos}

\section{Spot on line}

Son formatos y tecnologías específicas usadas por cada soporte para mostrar spots en Internet desde su sitio web. Todos los formatos estandarizados por IAB Spain son susceptibles de llevar un spot. 
Tabla 16

Área de superficie del spot on line

\begin{tabular}{|l|c|c|c|c|c|}
\hline \multicolumn{1}{|c|}{ Área píxeles } & $\begin{array}{c}\text { Bebidas } \\
\text { alcohólicas }\end{array}$ & Financiero & Telefonía & Moda & Total \\
\hline $\mathbf{1 4 0} \times \mathbf{4 0 0}$ & 1 & & & & 1 \\
\hline $\mathbf{1 5 0} \times \mathbf{1 2 2}$ & & & & 1 & 1 \\
\hline $\mathbf{1 6 0} \times \mathbf{6 0 0}$ & & & & 1 & 1 \\
\hline $\mathbf{1 8 0} \times \mathbf{1 9 0}$ & 1 & & & & 1 \\
\hline $\mathbf{3 0 0} \times \mathbf{2 5 0}$ & 2 & & & 1 & 3 \\
\hline Total & 4 & & & 3 & 7 \\
\hline
\end{tabular}

En total se han encontrado 7 spots on line, en un caso el formato corresponde al tamaño del banner y en tres casos al área estandarizada para los rascacielos. En general, cualquier formato es susceptible de incluir un spot.

\section{Patrocinio}

Para patrocinar un site, se suelen emplear formatos estándar como banners o botones. A menudo lindan hacia actividades off line, pudiendo actuar como puente entre los contenidos editoriales y comerciales.

Sólo se ha localizado un patrocinio cuyas medidas corresponden a $250 \times 90$ píxeles, que no corresponde con ninguno de los formatos estandarizados por IAB Spain. Su formato se aproxima al área de algunos robapáginas y botones.

\section{Рор ир}

Los formatos рор up aparecen sobre los contenidos o las páginas web, pudiendo dar sensación de narratividad a los anuncios. Sus dimensiones pueden ser $300 \times 250,200 \times 200$ y $250 \times 250$ píxeles. Usan la tecnología gif, animado o flash. Su peso es de $20 \mathrm{k}$.

No se ha encontrado ninguna ventan pop up en el análisis descriptivo de las campañas.

\section{Pop under}

Los formatos pop under aparecen debajo del navegador, es decir, de la página web que se está visitando. Pueden usar tecnología gif, animado o flash, entre otras. Sus dimensiones son $300 \times 250$ y su peso $25 \mathrm{k}$.

No se ha detectado ningún formato de este tipo en las creatividades analizadas. 


\subsection{Características referentes al contenido}

La intención del anunciante a través de la publicidad en la red es conseguir captar la atención de los navegantes. Para ello se hace un uso destacado de diferentes recursos expresivos como el empleo de ilustraciones, uso del color y la tipografía, utilización de sonidos, así como el contenido propiamente dicho de la creatividad.

En primer lugar, la publicidad en Internet se emplea, fundamentalmente, para el lanzamiento de los productos o servicios $(61,4 \%)$, tendencia más agudizada en el caso de la telefonía $(87,8 \%)$ debido, fundamentalmente, a las posibilidades de innovación propias del sector. En el caso del sector financiero las campañas prácticamente se reparten entre campañas de lanzamiento o mantenimiento, estrechamente relacionado con una mayor permanencia de los servicios que ofrece este ámbito.

Como cabía esperar, las campañas de expectación o teaser son muy poco frecuentes en la red debido a la menor capacidad que este el medio ofrece para seguir continuadamente la publicidad que en él se emiten.

Por otro lado, son infrecuentes los casos en los que hay más de una marca en la misma creatividad. Sólo en el 10,7\% de las piezas analizadas aparecen dos o más marcas relacionadas.

Tabla 17

Tipo de campaña y marca asociada a otra

\begin{tabular}{|l|c|c|c|c|c|}
\hline \multicolumn{1}{|c|}{ Tipo campaña } & $\begin{array}{c}\text { Bebidas } \\
\text { alcohólicas }\end{array}$ & Financiero & Telefonía & Moda & Total \\
\hline Lanzamiento & 63,4 & 50,3 & 87,8 & 62,5 & $\mathbf{6 1 , 4}$ \\
\hline Mantenimiento & 36,6 & 49,2 & 11 & 36,1 & $\mathbf{3 7 , 9}$ \\
\hline Teaser & - & 0,5 & 1,2 & 1,4 &, 7 \\
\hline Marca asociada a otra & & & & & \\
\hline No aparece & 85,9 & 92,9 & 81,7 & 91,7 & $\mathbf{8 9 , 3}$ \\
\hline Sí aparece & 14,1 & 7,1 & 18,3 & 8,3 & $\mathbf{1 0 , 7}$ \\
\hline
\end{tabular}




\subsubsection{Recursos gráficos y sonoros}

El $84,8 \%$ de los anuncios poseen ilustraciones, prácticamente la totalidad de las piezas de los sectores de bebidas alcohólicas y telefonía. En cuanto al tipo de ilustración, en la mayoría de los casos (60,6\%) estas ilustraciones son fotografías, tendencia más acusada en el sector de la telefonía (81\%) y de la moda $(78,3 \%)$. En el sector bancario las fotografías y los dibujos prácticamente aparecen equiparados en cuanto a su grado de utilización. Por otro lado, alrededor de un 10,6\% utilizan tanto fotografías como dibujos. Sin embargo, llama la atención que otro tipo de recursos expresivos que se pueden incorporar fácilmente a través de la tecnología rich media como la utilización del vídeo, prácticamente, no se estén utilizando por los anunciantes.

Tabla 18

Uso y tipo de ilustración

\begin{tabular}{|l|c|c|c|c|c|}
\hline \multicolumn{1}{|c|}{ Ilustración } & $\begin{array}{c}\text { Bebidas } \\
\text { alcohólicas }\end{array}$ & Financiero & Telefonía & Moda & Total \\
\hline Sí tiene ilustración & 98,6 & 75,1 & 96,3 & 84,7 & $\mathbf{8 4 , 8}$ \\
\hline No tiene ilustración & 1,4 & 24,9 & 3,7 & 15,3 & $\mathbf{1 5 , 2}$ \\
\hline Tipo de ilustración & & & & & \\
\hline Fotografía & 48,6 & 48,3 & 81,0 & 78,3 & $\mathbf{6 0 , 6}$ \\
\hline Dibujo & 27,1 & 40,3 & 8,9 & 15,0 & $\mathbf{2 6 , 5}$ \\
\hline Fotografía y dibujo & 18,6 & 11,4 & 8,9 & 1,7 & $\mathbf{1 0 , 6}$ \\
\hline Vídeo & 5,7 & - & 1,3 & 5,0 & $\mathbf{2 , 2}$ \\
\hline
\end{tabular}

Por otro lado, más de la mitad del total de las creatividades analizadas (54,5\%) poseen como elemento central de la ilustración un objeto, excepto en el sector de la moda, donde el elemento principal de la parte gráfica es una persona $(66,7 \%)$. En el resto de los sectores la aparición de una persona se encuentra en segundo lugar. Este comportamiento se reproduce en el caso del análisis de los elementos secundarios de las ilustraciones, excepto en el caso de la telefonía donde el 60,8\% de las creatividades que poseen una ilustración secundaria representan la imagen de una animal.

Además es muy poco frecuente la aparición como elementos centrales o secundarios de las imágenes de paisajes, rurales o urbanos, animales, edificios o imágenes abstractas. Como conclusión, las creatividades tratan de representar el producto anunciado o al usuario/cliente con el que se desea que se identifique el navegante. 
Tabla 19

Elemento principal y secundario de la ilustración

\begin{tabular}{|l|c|c|c|c|c|}
\hline $\begin{array}{c}\text { Elemento principal } \\
\text { ilustración }\end{array}$ & $\begin{array}{c}\text { Bebidas } \\
\text { alcohólicas }\end{array}$ & Financiero & Telefonía & Moda & Total \\
\hline Objeto & 44,3 & 61,1 & 72,2 & 26,7 & $\mathbf{5 4 , 5}$ \\
\hline Persona & 31,4 & 24,8 & 22,8 & 66,7 & $\mathbf{3 2 , 7}$ \\
\hline Paisaje rural & 12,9 & 6,7 & - & 3,3 & $\mathbf{5 , 9}$ \\
\hline Abstracto & 11,4 & 2,7 & 2,5 & 1,7 & $\mathbf{4 , 2}$ \\
\hline Edificio & - & 2,0 & - & 1,7 & $\mathbf{1 , 1}$ \\
\hline Animal & - & 2,0 & - & - & $\mathbf{8}$ \\
\hline Paisaje urbano & - &, 7 & 2,5 & - & $\mathbf{8}$ \\
\hline Elemento ilustración & & & & & \\
\hline secundaria & & & - & 35,0 & $\mathbf{5 1 , 1}$ \\
\hline Objeto & 35,7 & 59,7 & 15,2 & 26,7 & $\mathbf{1 8 , 7}$ \\
\hline Paisaje rural & 14,3 & 19,5 & 5,1 & 16,7 & $\mathbf{1 1 , 2}$ \\
\hline Abstracto & 21,4 & 7,4 & 11,4 & 8,3 & $\mathbf{9 , 5}$ \\
\hline Paisaje urbano & 17,1 & 5,4 & 7,6 & 10,0 & $\mathbf{6 , 4}$ \\
\hline Animal & 11,4 & 2,0 & 60,8 & 1,7 & $\mathbf{2 , 2}$ \\
\hline Edificio & - & 4,7 & - & 1,7 & $\mathbf{, 8}$ \\
\hline
\end{tabular}

Por otro lado, la mayoría de las creatividades utiliza tres colores diferentes (62,3\%), los más empleados son el azul, el rojo y naranja. Por sectores, las bebidas alcohólicas emplean con más frecuencia los colores rojo y negro, tonalidades con claras alusiones a la noche y al erotismo, que están directamente relacionados con las connotaciones que se asocian a este tipo de productos. En el sector financiero predomina la utilización del azul y blanco, fuertemente ligados a la imagen corporativa de muchas instituciones financieras, y en el caso del naranja ya que es el color corporativo del principal anunciante de este sector ING Direct. Respecto al sector moda, los colores más utilizados son el negro, signo de distinción y elegancia, azul y rojo, más ligado a ropa y complementos deportivos. 
Tabla 20

Número de colores y color predominante

\begin{tabular}{|l|c|c|c|c|c|}
\hline \multicolumn{1}{|c|}{ Número de colores } & $\begin{array}{c}\text { Bebidas } \\
\text { alcohólicas }\end{array}$ & Financiero & Telefonía & Moda & Total \\
\hline Tres & 77,5 & 62,9 & 54,9 & 54,2 & $\mathbf{6 2 , 3}$ \\
\hline Dos & 5,6 & 23,9 & 20,7 & 27,8 & $\mathbf{2 0 , 9}$ \\
\hline Cuatro & 16,9 & 10,2 & 19,5 & 12,5 & $\mathbf{1 3 , 5}$ \\
\hline Cinco & - & 3 & 3,7 & 1,4 & $\mathbf{2 , 4}$ \\
\hline Seis & - & - & - & 2,8 & $\mathbf{5}$ \\
\hline Nueve & - & - & 1,9 & - &, $\mathbf{2}$ \\
\hline Doce & - & - & - & 1,4 &, $\mathbf{2}$ \\
\hline Color predominante & & & & & $\mathbf{2 1 , 1}$ \\
\hline Azul & 5,6 & 25,9 & 25,6 & 18,1 & $\mathbf{1 5 , 6}$ \\
\hline Rojo & 32,4 & 12,7 & 9,8 & 13,9 & $\mathbf{1 5 , 2}$ \\
\hline Naranja & - & 24,9 & 7,3 & 4,2 & $\mathbf{1 2 , 3}$ \\
\hline Negro & 25,4 & 1,5 & 17,1 & 23,6 & $\mathbf{1 2 , 1}$ \\
\hline Blanco & 1,4 & 13,7 & 20,7 & 8,3 & $\mathbf{7 , 3}$ \\
\hline Verde & 11,3 & 8,6 & 2,4 & 5,6 & $\mathbf{6 , 9}$ \\
\hline Amarillo & 12,7 & 6,6 & - & 9,7 & $\mathbf{5 , 5}$ \\
\hline Gris & 1,4 & 3 & 14,6 & 5,6 & $\mathbf{1 , 2}$ \\
\hline Marrón & - & 1 & - & 4,2 & $\mathbf{1 , 2}$ \\
\hline Morado & - & 1,5 & 2,4 & - & $\mathbf{1 , 2}$ \\
\hline Rosa & 1,4 & 0,5 & - & 4,2 & \\
\hline
\end{tabular}

Si bien el color es un recurso directamente empleado para atraer la atención del usuario sobre la publicidad, no lo es tanto el empleo de una tipografía destacada -uso de negritas, diferentes cuerpos de letra, etc.- a diferencia de lo que ocurre en los medios gráficos. De esta manera sólo el $28,2 \%$ del total de las creatividades emplean una tipografía destacada, excepto en el sector financiero donde el 92,9\% de las creatividades hacen uso de los recursos tipográficos para llamar la atención sobre sus mensajes, probablemente porque esta publicidad es más informativa y da más importancia el aspecto textual, frente al resto de las creatividades de los otros sectores que están basadas en la atracción que ejerce el mensaje gráfico. 
Tabla 21

Uso de la tipografía

\begin{tabular}{|l|c|c|c|c|c|}
\hline \multicolumn{1}{|c|}{ Tipografía } & $\begin{array}{c}\text { Bebidas } \\
\text { alcohólicas }\end{array}$ & Financiero & Telefonía & Moda & Total \\
\hline Normal & 92,4 & 7,1 & 61 & 73,6 & $\mathbf{7 1 , 8}$ \\
\hline Destacada & 7,6 & 92,9 & 39 & 26,4 & $\mathbf{2 8 , 2}$ \\
\hline
\end{tabular}

Como se ha puesto de manifiesto con anterioridad, en la publicidad en Internet no se están aprovechando aún todas las posibilidades tecnológicas que ofrece el soporte. De esta manera sólo un 3,6\% de las creatividades disponen de sonido. De las creatividades que disponen de sonido, el $40 \%$ acompañan los elementos gráficos con música y el $100 \%$ en bebidas alcohólicas. Una explicación a esta ausencia de sonido puede ser que los anunciantes prefieren optar por una publicidad menos intrusiva, que interfiera menos en la navegación del usuario.

Tabla 22

Uso del sonido y tipo de sonido

\begin{tabular}{|l|c|c|c|c|c|}
\hline \multicolumn{1}{|c|}{ Sonido } & $\begin{array}{c}\text { Bebidas } \\
\text { alcohólicas }\end{array}$ & Financiero & Telefonía & Moda & Total \\
\hline Sí tiene sonido & 11,3 & 1 & 3,7 & 2,8 & 3,6 \\
\hline No tiene sonido & 88,7 & 99 & 96,3 & 97,2 & 96,4 \\
\hline Tipo de sonido & & & & & \\
\hline Música & 37,5 & 100 & - & 50 & 40 \\
\hline Canción & 37,5 & - & - & - & 20 \\
\hline Voz & 25 & - & - & - & 13,3 \\
\hline Voz y música & - & - & 100 & 50 & 26,7 \\
\hline
\end{tabular}

\subsubsection{Recursos referentes al contenido de la creatividad}

Si bien en la publicidad gráfica a través de otros medios convencionales es muy frecuente la utilización de un eslogan para facilitar la memorización del producto, en la publicidad en Internet sólo en un $38,2 \%$ de las creatividades aparece un eslogan relacionado con la marca. Sin embargo, en el sector financiero y de telefonía se reparten casi a partes iguales las creatividades que disponen de un eslogan frente a las que no lo tienen. Por otro lado, en el 91,9\% de las piezas publicitarias aparece el logotipo de la marca como elemento clave para facilitar la identificación de la pieza publicitaria con el anunciante. 
Tabla 23

Uso del eslogan

\begin{tabular}{|l|c|c|c|c|c|}
\hline \multicolumn{1}{|c|}{ Eslogan } & $\begin{array}{c}\text { Bebidas } \\
\text { alcohólicas }\end{array}$ & Financiero & Telefonía & Moda & Total \\
\hline No tiene & 70,4 & 59,9 & 52,4 & 69,4 & $\mathbf{6 1 , 8}$ \\
\hline Sí tiene & 29,6 & 40,1 & 47,6 & 30,6 & $\mathbf{3 8 , 2}$ \\
\hline Logotipo & & & & & \\
\hline Sí tiene & 91,5 & 90,9 & 90,2 & 97,2 & $\mathbf{9 1 , 9}$ \\
\hline No tiene & 8,5 & 9,1 & 9,8 & 2,8 & $\mathbf{8 , 1}$ \\
\hline
\end{tabular}

Respecto al idioma utilizado, el $87,2 \%$ están expresadas en castellano. Se aprecia una mayor utilización del inglés en el sector moda $(22,2 \%)$ y telefonía $(12,2 \%)$, mientras en el sector financiero y de bebidas alcohólicas no se emplean idiomas extranjeros. Es prácticamente anecdótico el uso del francés, de igual manera que en sólo un $5 \%$ de los casos analizados se hace usos de otras lenguas oficiales en España.

Tabla 24

Uso del idioma

\begin{tabular}{|l|c|c|c|c|c|}
\hline \multicolumn{1}{|c|}{ Idioma } & $\begin{array}{c}\text { Bebidas } \\
\text { alcohólicas }\end{array}$ & Financiero & Telefonía & Moda & Total \\
\hline Castellano & 91,5 & 94,9 & 76,8 & 73,6 & $\mathbf{8 7 , 2}$ \\
\hline Inglés & - & - & 12,2 & 22,2 & $\mathbf{6 , 2}$ \\
\hline $\begin{array}{l}\text { Otras lenguas } \\
\text { oficiales españolas }\end{array}$ & 8,5 & 5,1 & 3,7 & 2,8 & $\mathbf{5}$ \\
\hline Francés & - & - & 4,9 & 1,4 & $\mathbf{1 , 2}$ \\
\hline Otros idiomas & - & - & 2,4 & - & $\mathbf{5}$ \\
\hline
\end{tabular}

Sólo el 28\% de las creatividades ofrecen los datos de contacto del anunciante. Este porcentaje es aún menor en el sector de la telefonía. Entre los datos que contacto que se ofrecen destaca el número de teléfono $(47,5 \%)$ y, en segundo lugar, la URL $(45,8 \%)$. Por otro lado, es casi anecdótica la aparición de la dirección postal del anunciante. El problema que podría ocasionar para el usuario la ausencia de estos datos de contacto aparece contrarrestado con el hecho de que en la mayoría de las piezas publicitarias existen enlaces que permiten al usuario recabar más información acerca del producto o servicio publicitado. 
Tabla 25

Uso y tipo de datos de contacto

\begin{tabular}{|l|c|c|c|c|c|}
\hline \multicolumn{1}{|c|}{ Datos de contacto } & $\begin{array}{c}\text { Bebidas } \\
\text { alcohólicas }\end{array}$ & Financiero & Telefonía & Moda & Total \\
\hline Tiene datos & 26,8 & 37,6 & 8,5 & 25 & 28 \\
\hline No tiene datos & 73,2 & 62,4 & 91,5 & 75 & 72 \\
\hline Tipo de datos & & & & & \\
\hline URL & 100 & 21,6 & 28,6 & 94,4 & 45,8 \\
\hline Teléfono y URL & - & 6,8 & 14,3 & 5,6 & 5,9 \\
\hline Teléfono & - & 71,6 & 42,9 & - & 47,5 \\
\hline Dirección postal & - & - & 14,3 & - & 0,8 \\
\hline
\end{tabular}

Frente al contenido de la publicidad en los inicios de Internet que utilizaban como recursos para llamar la atención del navegante la formulación de preguntas, adivinanzas, etc., para conseguir que el usuario hiciera "clic" sobre la publicidad (AUC, 2000; Chandon, Chtourou y Fortín, 2003), la tendencia en la actualidad es hacia una menor utilización de estas técnicas. De esta manera, sólo un 11,6\% formula una pregunta al navegante y únicamente un 16,1\% reclama de una manera explícita que el usuario realice clic sobre la publicidad. Por otro lado, también se aprecia por parte de los anunciantes un mayor interés por no defraudar las expectativas del usuario -el $97,4 \%$ de la publicidad analizada y $100 \%$ en sector financiero-, es decir, proporciona la información que el navegante reclama sin utilizar artificios que conduzcan al usuario a otras páginas no seleccionadas en su navegación.

Tabla 26

Formula una pregunta, reclama "clic" y respeta las expectativas del navegante

\begin{tabular}{|l|c|c|c|c|c|}
\hline \multicolumn{1}{|c|}{ Formula pregunta } & $\begin{array}{c}\text { Bebidas } \\
\text { alcohólicas }\end{array}$ & Financiero & Telefonía & Moda & Total \\
\hline No formula & 58,8 & 90,4 & 82,9 & 95,8 & $\mathbf{8 8 , 4}$ \\
\hline Sí formula & 18,3 & 9,6 & 17,1 & 4,2 & $\mathbf{1 1 , 6}$ \\
\hline Reclama clic & & & & & \\
\hline No reclama & 87,3 & 83,8 & 85,4 & 79,2 & $\mathbf{8 3 , 9}$ \\
\hline Sí reclama & 12,7 & 16,2 & 14,6 & 20,8 & $\mathbf{1 6 , 1}$ \\
\hline Respeta deseo usuario & & & & & \\
\hline Sí lo respeta & 93 & 100 & 96,3 & 95,8 & $\mathbf{9 7 , 4}$ \\
\hline No lo respeta & 7 & - & 3,7 & 4,2 & $\mathbf{2 , 6}$ \\
\hline
\end{tabular}




\section{Conclusiones}

Internet ofrece muchas posibilidades para la publicidad que aún no se están aprovechando. Entre las principales conclusiones del estudio destaca la utilización predominante de la tecnología rich media y la falta de estandarización de los formatos publicitarios.

Respecto al empleo de la tecnología rich media, las aplicaciones flash y gif animado son las más empleadas por los anunciantes. Por este motivo predominan las imágenes dinámicas frente a las estáticas, aunque se está haciendo un uso muy limitado y escaso de otras posibilidades que ofrece esta tecnología como la inclusión en la creatividad de efectos de sonido o imágenes animadas. Por otro lado, es llamativo el hecho de que el $90,5 \%$ de las creatividades no sean interactivas, aunque en la mayoría aparece un enlace a través del cual el navegante puede acceder a más información sobre el producto o servicio publicitado.

En cuanto a la diversidad de tamaños y formatos, hay que dejar claro que la ausencia de estandarización de los formatos no repercute en el receptor ya que visualmente la diferencia de tamaño no afecta al reconocimiento de la forma publicitaria ante la que se encuentra. Sin embargo, sí supone un gran problema a la hora de realizar la creatividad y, sobre todo, la planificación de medios.

La disparidad de tamaños es más que evidente, sólo hay que observar que coexisten un total de 87 tamaños frente a los 18 propuestos por IAB Spain. Por esta razón se impone la necesidad de unificar los tamaños de las creatividades lo que, sin duda, facilitará la planificación y diseño de la creatividad en este soporte. Por otro lado, también se pone de manifiesto cómo el formato que se ha utilizado más frecuentemente y desde los inicios de la publicidad en este soporte, el banner, es la forma publicitaria más estandarizada (IAB, 200), así el 73,5\% utilizan el área propuesta por IAB Spain. Aunque aún queda mucho por hacer ya que se han detectado un total de 26 tamaños diferentes que visualmente se reconocen como banner. Al mismo tiempo, prácticamente todas las creatividades de otro de los formatos más empleados, el robapágina, responden a los formatos estandarizados por IAB Spain (78,7\%).

Respecto a los rascacielos, el 35,5\% presentan el tamaño recomendado por IAB Spain. A pesar de que no se emplean estos tamaños estandarizados es el formato en el que las medidas, en general, respecto a los píxeles horizontales y verticales presentan una mayor uniformidad.

Pero si hay un formato en el no se ha conseguido que se respeten los estándares es precisamente en el caso del botón. En total se han encontrado 49 áreas diferentes, de las que sólo una corresponde a una de las medidas estándar de IAB Spain. Quizá esta situación se deba a que los botones son las piezas publicitarias que permiten una mayor flexibilidad, lo que dificulta el llegar a un consenso sobre cuál tiene que ser el formato estándar.

En cuanto al resto de los formatos, es difícil establecer si se está consiguiendo una estandarización debido al escaso número de piezas publicitarias encontradas. Sin embargo, tomando con mucha cautela 
los datos, podemos concluir que tampoco se ha logrado establecer un estándar respecto al layer, interstitial, spot on line o patrocinio.

Otra conclusión del estudio apunta a que se intenta realizar una publicidad menos intrusiva. Prueba de ello es que se está optando por los formatos considerados menos molestos por los usuarios como el banner, rascacielos, robapáginas o botón. Al mismo tiempo que se utilizan muy poco los formatos que más interfieren en la navegación del usuario como los layers.

En este sentido, la publicidad es más respetuosa con el usuario que antes (AUC, 1999) como pone de manifiesto el hecho de que los anuncios no reclamen clic y no hagan preguntas directamente al usuario. Al mismo tiempo que se intenta respetar las expectativas que tiene el navegante sobre la publicidad a la que presta atención.

Respecto a los recursos expresivos, en la mayoría de los anuncios aparecen ilustraciones, preferentemente fotografías cuyos elementos principales son objetos o personas que representan al producto anunciado o al cliente potencial con el que el anunciante quiere que se identifique el navegante.

En cuanto al uso del color, se utilizan entre dos y cuatro colores, preferentemente tres. Los más empleados son el azul, que coincide con el color corporativo de grandes anunciantes, rojo y naranja, colores cálidos, atractivos dada su capacidad para llamar la atención y que en el caso del color naranja también coincide con el color corporativo de algunos anunciantes. En cuanto al uso de una tipografía destacada, ésta es más importante en el caso de la publicidad de tipo informativo, propia de productos funcionales como los financieros frente a la utilizada en el caso de productos expresivos.

Por otro lado, se hace poco uso del eslogan pero sí se recurre mucho el logotipo como elemento de identificación. Al mismo tiempo que no suelen aparecer los datos de contacto del anunciante, aunque en algunas ocasiones se reseña el número de teléfono o la URL. En último lugar, la inmensa mayoría de las creatividades están redactadas en castellano.

Respecto a la ubicación de la publicidad se manifiesta una tendencia hacia la rotación general dentro del conjunto de la página y a la existencia de más publicidad en los soportes que proporcionan contenidos de interés general como información y noticias y portales y buscadores.

Internet se utiliza como soporte para dar a conocer determinados productos o servicios, así la mayoría de las campañas analizadas son de lanzamiento del producto. Los artículos más anunciados son los servicios financieros, teléfonos móviles y moda; productos directamente relacionados con las características propias de este soporte publicitario, fundamentalmente informativo y muy ligado al concepto de innovación y actualidad.

Por tanto, uno de los principales dilemas a los que se enfrenta la publicidad en Internet es resolver el problema de la diversidad de tamaños de los formatos. Por esta razón se impone la necesidad de 
unificar el área de superficie de las creatividades, lo que sin duda facilitará la planificación y el diseño de la creatividad en este soporte.

En cuanto a las limitaciones de este estudio, sería recomendable ampliar el análisis de las creatividades a otros sectores y añadir otras especificaciones del formato como el peso de la creatividad, que es fundamental a la hora de insertarla en la página web.

\section{Referencias bibliográficas}

- Asociación de Usuarios de la Comunicación (1999). Análisis de la publicidad en Internet. Informe 1999. Extraído el 1 de diciembre, 2005 de http://www.auc.es.

- Cho, C.H., Lee, J. y Tharp, M. (2001). Different Forced-Exposure Levels to Banner Advertisements. Journal of Advertising Research. 41 (4) 45-56.

- Chandon, J. L.; Chtourou, M. S. y Fortin, D. R. (2003). Effects of Configuration and Exposure levels on Responses to Web Advertisements. Journal of Advertising Research, June 217-229

- DoubleClick (2005). The evolution or Rich Media Advertising: Currente market trands, success metrics and best practices. Extraído el 1 de diciembre, 2005 de http:// www.doubleclick.com/knowledge)

- Frutos Torres, B. y Gutiérrez Zotes, P. (2005). Respuestas congnitivas de los usuarios a los contenidos publicitarios en "rich media”. X Congreso Internet, Telecomunicaciones y Sociedad de la Información. Madrid: Mundo Internet.

- Frutos Torres, B y Sánchez Valle, M. (en prensa). La eficacia del formato y la animación en la publicidad en Internet iSe ha desgastado el banner?

- Hoffman D.L. and Novak, T. P. (1997). New Metrics for New Media: Toward the Development of Web Measurement Standars. World Wild Web Journal, 2,1.

- IAB Europe (2002). Introducing the creative formats for interactive advertising. Extraído el 31 de febrero, 2006 de URL: http//:www.iab.es

- IAB Spain (2003). Formatos creativos estandarizados por el IAB hasta 2003. Extraído el 1 de febrero, $2006 \mathrm{de} \mathrm{http//:www.iab.es}$

- IAB Spain (2004). Formatos estandar de la publicidad interactiva 2004. Extraído el 1 de febrero, 2006 de URL: http//:www.iab.es

- IAB y PricewatherhouseCoopers (2005). Estudio sobre la inversión publicitaria en medios interactivos. Interactive Advertising Bureau, Spain. Extraído el 1 de diciembre, 2005 de URL: http//:www.iab.es 
- INFOADEX (2005): La inversión publicitaria en España 2005. Extraído el 1 de mayo, 2005 de URL: http//: www.infoadex.es

- INFOADEX (2006): La inversión publicitaria en España 2006. Extraído el 1 de marzo, 2005 de http//: www.infoadex.es

- Meland (2000, Febrero). Banner Advertisements Get Sexy. Forbes.com. Extraído el 1 de marzo, 2005 de http//:www.forbes.com 